JOURNAL DE PHYSIQUE IV

Colloque C4, supplément au Journal de Physique III, Vol. 1, novembre 1991

\title{
TRANSFORMATION BEHAVIOUR OF THE Al - 65\% Ni ALLOY
}

\author{
J.H. MAAS, L. TOTH(1), A.A.H. HAMERS and J. BEYER \\ University of Twente, Faculty of Mechanical Engineering, Laboratory for Materials Science, PO. Box \\ 217, NL-7500 AE Enschede, The Netherlands
}

\begin{abstract}
The martensitic transformation and the precipitation behaviour of quenched Ni6sAl35 alloys were studied by structural and thermal analytical techniques. It is shown that the thermally induced martensite phase is not thermoelastic at this composition, but thermoelastic martensite may be created by the introduction of thermal or mechanical stress. The NisAls precipitate phase developed from the martensite during annealing above $600 \mathrm{~K}$ is suggested to be metastable, in contrast to the generally accepted view. The presence of NizAl type ordering in the martensite phase of slowly cooled samples is reported and correlated with the greater stability and irreversibility of the martensite.
\end{abstract}

\section{Introduction}

The very strong interaction between the $\mathrm{Ni}$ and $\mathrm{Al}$ atoms plays an important role in the formation of most phases of the Ni-Al system. A series of stable or metastable phases has been observed in the $\beta$-phase region which has been reviewed recently by Locci et al [1]. The formation of these phases has been related to short range order of the excess $\mathrm{Ni}$ atoms on the Al sublattice of the ordered bcc NiAl lattice.

Like many of the $\beta$-phase alloys the Ni-rich NiAl (with about $62-66$ at\% Ni) is subject to a martensitic transformation which takes place either upon cooling (thermally induced martensite, TIM) or deformation (stress induced martensite, SIM) [2]. The thermoelastic nature of the martensite is recognised [3, 4] and the shape memory behavior is first demonstrated by Enami and Nenno [5]. The crystal structure of the tetragonal martensite phase has been first reported to be a modified L10 [6], but the authors of later studies generally found it to be the original (unmodified) Llo ordered structure $[2,3,7]$. The deformation mode of this transformation can be derived from the instability of the $\beta$-NiAl lattice against $\{110\}<1 \overline{10}\rangle-$ shear $[8]$. Chandrasekaran and coworkers $[9,10,11]$ reported several stacking modulated structures in the martensite phase in various NiAl alloys (e.g. Ni65Al35). The \{111\}t or $\{110\}$ B2 atomic planes were sheared systematically resulting in several observed stacking sequences $3 \mathrm{R}, 7 \mathrm{R}, 2 \mathrm{H}$ and others $(10 \mathrm{H})$ which they explained by the variation of the matrix-twin thickness ratio. The occurrence of the different structures has also been found to be composition dependent. Reynaud [12] as well as Enami et al [13] observed the seven-layer (7R) structure in the martensite and interpreted it as a premartensitic phase. The latter authors showed that the same amount of (111) [112]t type elementary shear of the neighbouring (111)t "close packed" planes can explain all structures observed by systematically varying the sense of shears. As pointed out by Martynov et al [2] the type and magnitude of this shear is the same as that of the twin boundaries commonly observed in the Llo (3R) martensite. They attributed a monoclinic elementary cell for both the $3 R$ and the $7 R$ modifications with different values for the angle $\beta$ and length $c$. The $7 R$ martensite can also be regarded as a $3 R$ stacking sequence with two twin boundaries arranged periodically in every seven

(1) Permanent address: Research Institute for Technical Physics, Budapest, Hungary 
layers and also denoted as (52) structure [2].

The above picture has been developed further by Tanner et a1 $[14,15,16]$ to inrlinde the structural development of the premartensitic phase. By combination of inelastic neutron scattering and high resolution $\mathrm{TEM}$ they identified an incomensurate modulated structure in NixAl1-x $(x=62.5-63$ at\%) well above the Ms point which turned out to be of the same $\{110\}<110>_{\mathrm{B} 2}$ type but of lesser amplitude than the shears for the $3 R$ or $7 R$ martensites.

Reynaud [17] observed an ordered superlattice in NixAl1-x samples ( $\quad-62.5$ at\%) annealed at 573 - $673 \mathrm{~K}$ for long time after slow cooling from $1423 \mathrm{~K}$ wich he attributed to short range ordering process leading to a hexagonal ordered phase of NizA1 composition. Others [18] stated this phase as metastable, but recently Locci et al [1] observed the same phase within the twins of $7 R$ martensite phase after aging 12 hour at $823 \mathrm{~K}$. Litvinov et al [19] observed another ordered structure consisting of 8 fct cells with the NisAl3 composition. Enami and Nenno [20] based the crystal lattice of their precipitated structure to be of the same composition but a different type of ordering (Pt5Gas structure) which can be formed from the martensite phase by a combination of diffusion and shuffle processes. This structure has also been confirmed by Robertson and Wayman [18] for samples with 63 and 70 at\% $\mathrm{Ni}$ aged at 773 - $973 \mathrm{~K}$. However it is worth to remark that:

(i) the above NisAl3 phase has been found to be most stable at around 66 at\% Ni instead of the stoichiometric 62.5 at\% on the contrary NizAl has been observed in samples near 62.5 at\% $\mathrm{Ni}$ content.

(ii) the precipitates have only been formed by quenching followed by aging and not by slow cooling suggesting a tempering of martensite. Despite of this fact the NisAl3 phase is recently considered as a stable one in the latest version of the equilibrium phase diagram [21].

The aim of the present paper is to draw the attention to how the various phase changes observed in NiAl during heat treatments are related to the $\beta$ or martensite phases and an effort is made to relate these changes to the different ways of ordering of the excess $\mathrm{Ni}$ atoms on the $\mathrm{Al}$ sublattice. This is supported by experiments carried out on both quenched and slowly cooled samples of nominal composition of Ni6sA135.

\section{Experimental}

Specimens of NiAl with nominal composition of 65 at\% $\mathrm{Ni}$ were prepared by arc melting under Argon atmosphere. The buttons of $20 \mathrm{~g}$ were melted several times to ensure homogeneity. The samples were helium quenched from $1523 \mathrm{~K}$ to room temperature (RT) in a quench dilatometer with a rate of about $100 \mathrm{k} / \mathrm{s}$. Standard differential scanning calorimetry (DSC, heating rate of $10 \mathrm{k} / \mathrm{min}$ ), dilatometry, X-ray diffraction (XRD), transmission electron microscopy (TEM) and optical microscopy were used as techniques for characterization of the samples.

Results and discussion

Optical micrographs of polished specimens quenched to RT show a martensite morphology (Fig. 1). Electron microscopy carried out on thin specimens confirmed that the martensite structure is face centered tetragonal (3R stacking sequence) with several variants coexisting. Within each variant a dense array of parallel planar twin boundaries have been observed. Our TEM observations, which were confirmed by X-ray diffraction, coincide with those published by Chandrasekaran [11] about the structure and morphology of the martensitic phase in a range of NiAl alloys.

During the first heating cycle of the quenched samples in the DSC generally four different type of processes are observed (Fig. 2).

The first endothermic peak (Fig.2) at $533 \mathrm{~K}$ which is frequently weak or even absent, can be assigned to the reversible martensite - austenite transformation. This means that the reversible martensite is confined to a small portion of the total volume. 


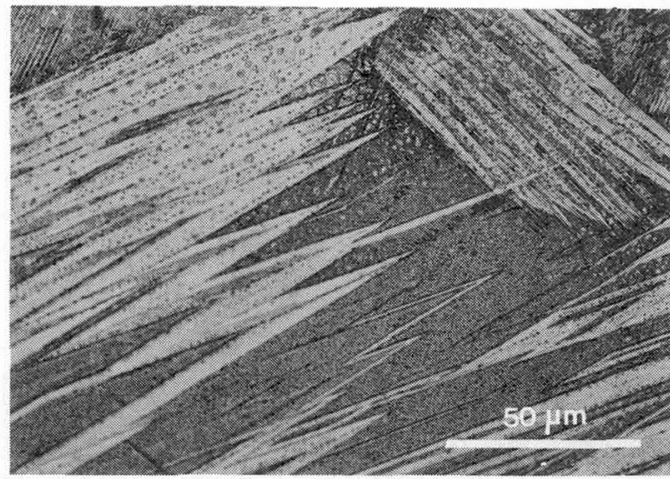

Fig. 1. Optical micrograph of martensite plates in as quenched Ni65Aliss etched by modified Kalling.

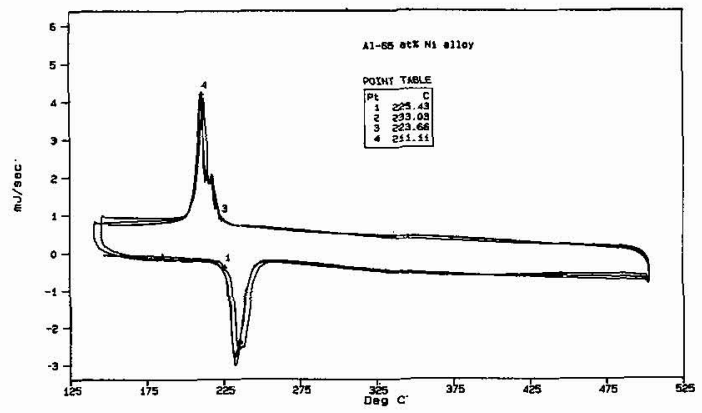

Fig. 3. Reversible martensitic transformation in DSC after mechanical grinding of an as quenched Ni65Al3s sample.

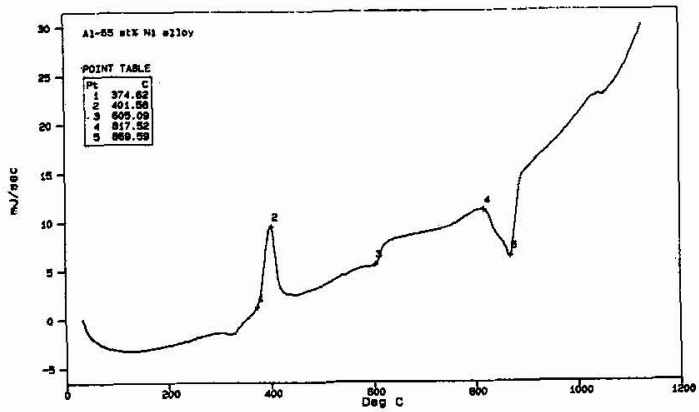

Fig. 2. Characteristic DSC diagram of the first heating up of as quenched Ni6sal3s samples showing peaks caused by four different processes.

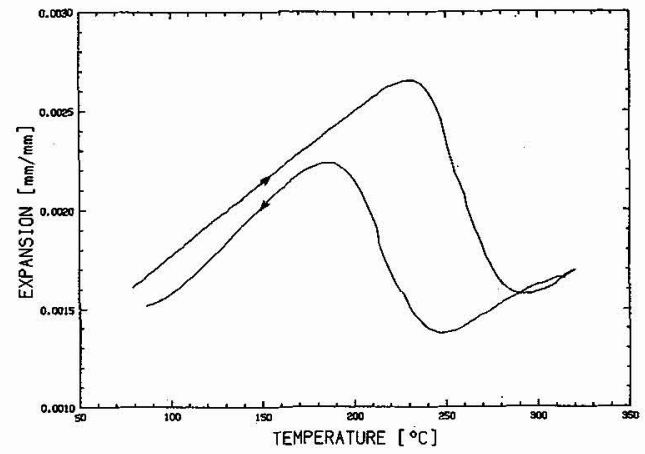

Fig. 4. Dilatometer evidence of reversible martensitic transformation in as-quenched Ni6sAl35 sample.

Manual grinding of as-quenched samples often resulted in a more pronounced martensite peak in the DSC which proved to be caused by the same reversible transformation (Fig.3). It is believed that the mechanical stress introduced during grinding is restricted to a rather thin surface layer thus modifying the $3 R$ martensite by stress: Similar reversible martensite transformation in as quenched NibsAl35 samples has been observed in the dilatometer (Fig.4). In this case the internal stress is supplied by the thermal gradient between the surface and bulk of the sample during quenching. It is believed that reversible (3R) martensite phases reported in the literature are caused by the same thermal or mechanical stresses as demonstrated here. The major volume of martensite, confirmed to be face centered tetragonal 3R in accordance with the literature data, is not reversible at this composition range of the quenched NiAl alloys. Stacking sequences, different from the $3 R$, with a period of 10 atomic 1 ayers were revealed in some parts of the sample whereas the repetition pattern of twin boundaries was irregular in neighbouring areas resulting in (111)t streaks in the twinned 3R diffraction pattern (Fig.5). Chandrasekaran observed similar ten layer (in addition to a 7 layer) period in splat cooled Ni63Al37 as the accommodation mode in the martensite [11]. In samples of similar composition Martynov [2] and Schryvers [22] conclude that the stress induced martensite is the $7 R$ modification. We assume therefore that in our samples the same $7 R$ stacking (or similar) formed from the martensite is responsible for the reversible transformation. 
Only the 3R martensite is subject to tempering as shown by the second large exothermic peak in the DSC (Fig. 2) above the Af temperature of the reversible martensite (around $623 \mathrm{~K}$ ). During this tempering the formation of the NisAl3 phase is confirmed by X-ray diffraction as well as by TEM. (Fig.6). After the precipitation of NisAl3 no martensite transformation is observed on repeated cycling in the DSC. We did not observe the NisAl3 phase during slow cooling in DSC or dilatometer, indicating that it only precipitates from the martensite. This is supported by the fact that after quenching to $673 \mathrm{~K}$ and keeping there for two days no $\mathrm{Ni} 5 \mathrm{Al} 3$ was observed by $\mathrm{X}$-ray diffraction. Therefore we raise some doubts about incorporating this phase in the equilibrium phase diagram.
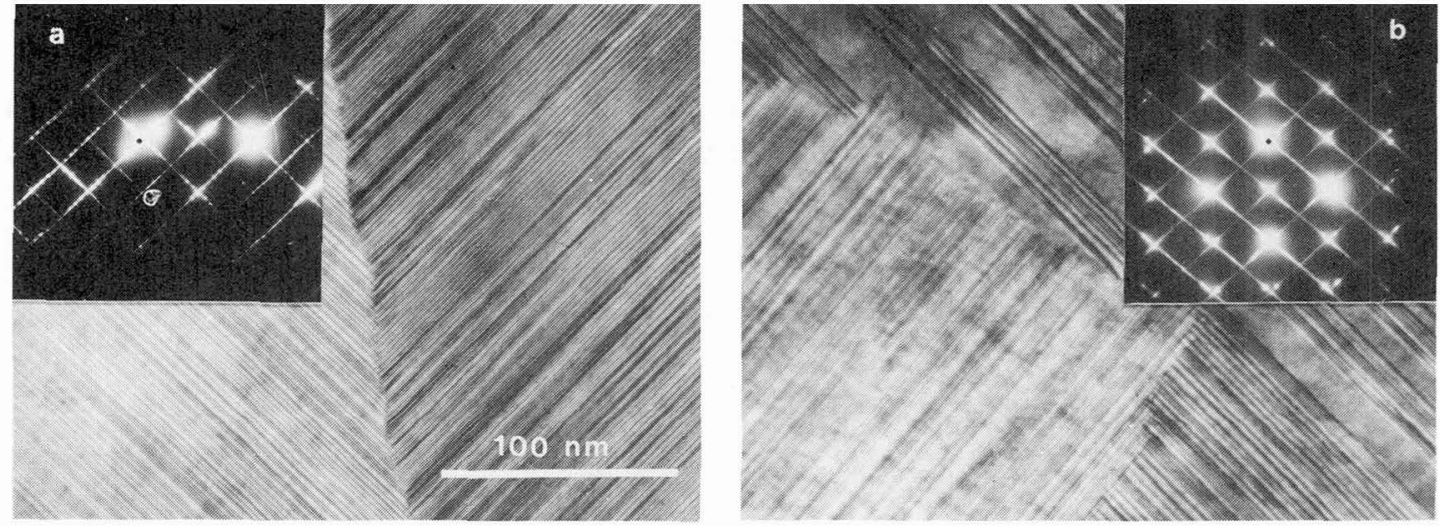

Fig. 5. TEM micrographs and ED patterns showing different microstructures in a single specimen of as-quenched Ni65Al35 martensite: (a) regular array of twin boundaries (ten-layer period) and (b) irregular (non-periodic) arrangement of twin boundaries.
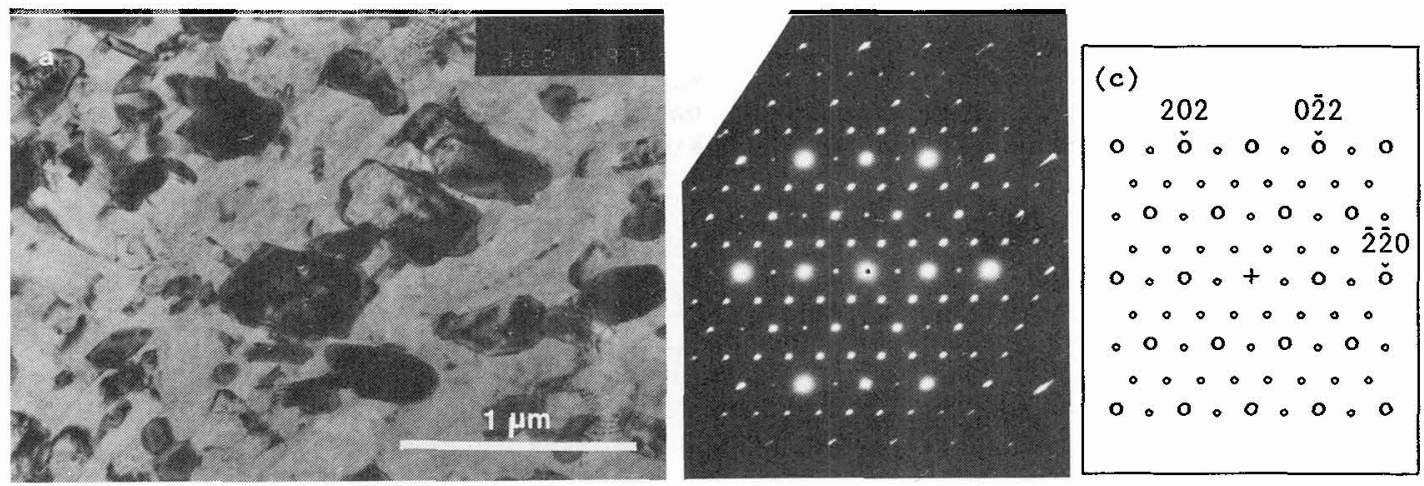

Fig. 6. TEM micrograph (a) and ED pattern (b) of $\mathrm{NisAl} 3$ phase formed during annealing in the electron microscope at $770 \mathrm{~K}$. The indexing (c) refers to the tetragonal (3R) martensite (large circles). Small circles denote NisAl3 superlattice reflections.

The third peak in Fig. 2 (an endothermic process at 773-823 K) is also weak and its size seems to be related to that of the reversible martensite peak. In addition this transition also showed to be reversible on cycling within the DSC (Fig. 7). We believe that this peak belongs to an order - disorder transition related to the formation of NizAl phase. This transition might be described as a sublattice ordering process causing clusters of Ni2Al to develop due to compositional variations. A 
Only the $3 R$ martensite is subject to tempering as shown by the second large exothermic peak in the DSC (Fig. 2) above the Af temperature of the reversible martensite (around $623 \mathrm{~K}$ ). During this tempering the formation of the NisAl3 phase is confirmed by X-ray diffraction as well as by TEM. (Fig.6). After the precipitation of NisAl3 no martensite transformation is observed on repeated cycling in the DSC. We did not observe the NisAls phase during slow cooling in DSC or dilatometer, indicating that it only precipitates from the martensite. This is supported by the fact that after quenching to $673 \mathrm{~K}$ and keeping there for two days no $\mathrm{Ni5A} / 3$ was observed by X-ray diffraction. Therefore we raise some doubts about incorporating this phase in the equilibrium phase diagram.
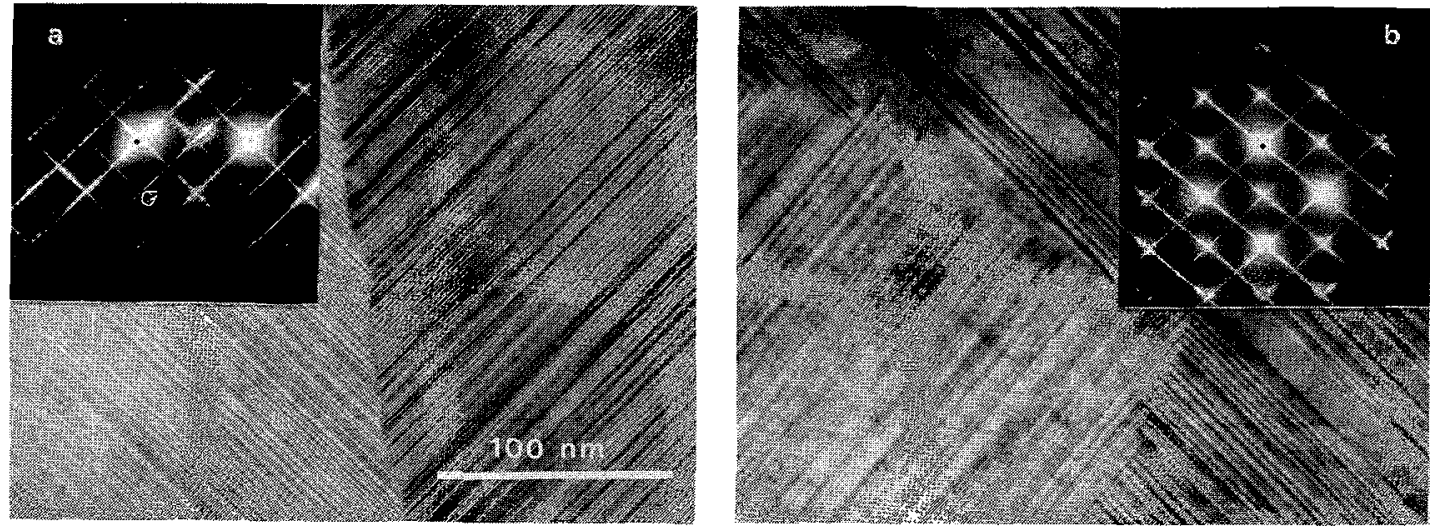

Fig. 5. TEM micrographs and ED patterns showing different microstructures in a single specimen of as-quenched Ni6sAl35 martensite: (a) regular array of twin boundaries (ten-layer period) and (b) irregular (non-periodic) arrangement of twin boundaries.
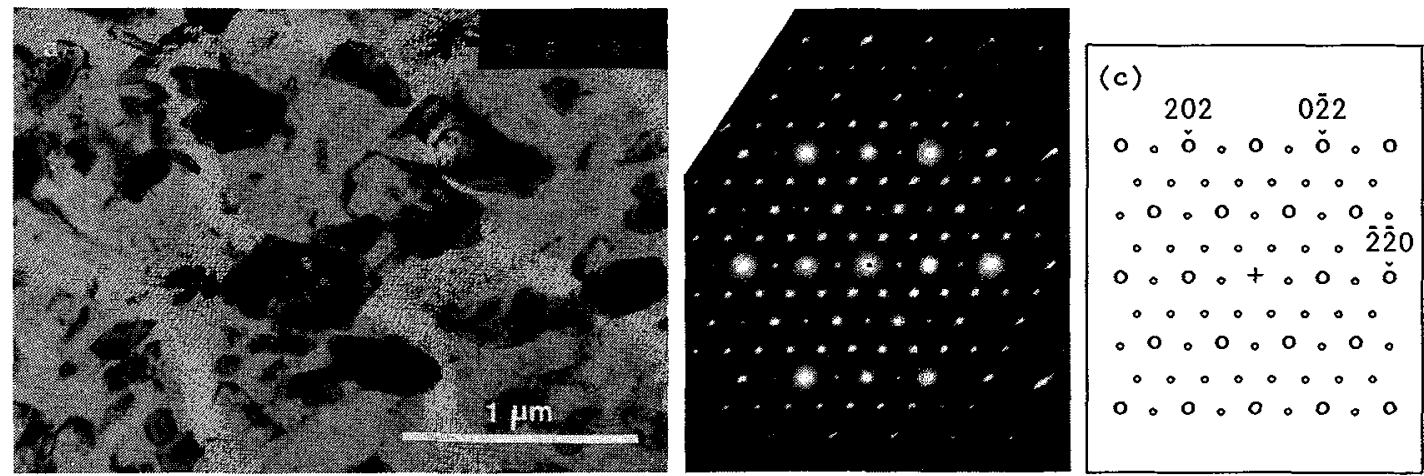

Fig. 6. TEM micrograph (a) and ED pattern (b) of NisAl3 phase formed during annealing in the electron microscope at $770 \mathrm{~K}$. The indexing (c) refers to the tetragonal (3R) martensite (large circles). Small circles denote NisAl3 superlattice reflections.

The third peak in Fig. 2 (an endothermic process at 773-823 K) is also weak and its size seems to be related to that of the reversible martensite peak. In addition this transition also showed to be reversible on cycling within the DSC (Fig. 7). We believe that this peak belongs to an order - disorder transition related to the formation of NizAl phase. This transition might be described as a sublattice ordering process causing clusters of Ni2Al to develop due to compositional variations. A 
similar mechanism was first proposed by Reynaud [12] for the formation of Ni2Al ordered phase observed within the $\beta$-phase of NixAl1-x $(x=62.5$ at $)$.

By means of TEM we have observed an ordered phase within the $3 R$ martensite phase in homogenized ( $1523 \mathrm{~K}$ ) and then furnace cooled NiasAl3s samples (Fig. 8). This ordering obviously took place in the $\beta$ phase but the topology of the atomic arrangement was inherited by the martensite phase. Therefore our electron diffraction patterns were only similar but not exactly the same as those of Reynaud [17]. An ordering of the Ni atoms on the Al sublattice in specific directions within the (110) planes of the $\beta$ phase might explain its reversibility and the fact that the presence of NizAl type ordering does not obstruct the martensite formation as is observed by DSC and electron microscopy. The Ms of these samples, however, is shifted to somewhat higher values than that of the reversible martensite indicating a destabilization effect of the $\beta$ phase by this ordering. Further research on the structure of this ordered phase is in progress and will be described elsewhere [23].

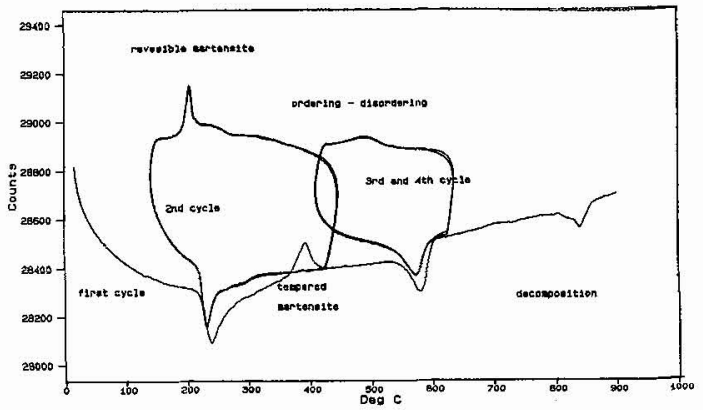

Fig. 7. DSC diagram of reversible order - disorder transition upon cycling between 673 and $873 \mathrm{~K}$.
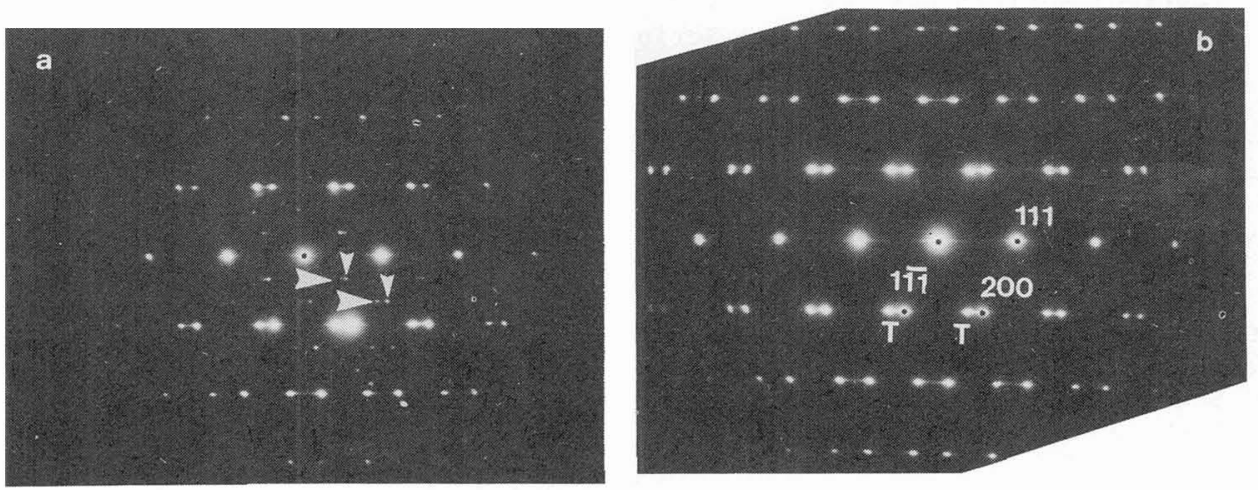

Fig. 8. [0ī1] t zone electron diffraction patterns of furnace cooled (a) and quenched (b) Ni65Al35 sample. Arrows indicate $1 / 3\{311\}$ and 1/3(022) type superlattice spots.

The fourth (endothermic) peak at $1073 \mathrm{~K}$ reflects the decomposition of the $\beta$ phase into the stable phases Ni3Al and $\beta$ with equilibrium concentration of $\mathrm{Ni}$. 


\section{Conclusions}

It has been shown that the thermally induced martensite phase in as-quenched Ni65Al35 is - as a rule - not reversible. Thermal or mechanical stress is a prerequisite for the reversible behaviour of the martensite.

The structure of the precipitate phase developed during annealing of thermally induced martensite above $600 \mathrm{~K}$ has been confirmed to be the NisAl3 phase, recently regarded as an equilibrium phase. However, we have shown arguments that it is a metastable phase which can only be formed from the martensite. It may therefore be regarded as a tempering effect of the martensite.

In the martensite a Ni2Al type ordering of the $\mathrm{Ni}$ atoms on the $\mathrm{Al}$ sublattice has been observed which had been inherited from the NizAl ordering in the $\beta$ phase. This phase has been developed in furnace cooled Ni65Al3s samples as well as in those quenched to $673 \mathrm{~K}$ and annealed there for two days. This ordering might contribute to a stabilization effect of the martensite thereby influencing its reversibility.

We have demonstrated with the available techniques some of the interrelations between the different phase transformations but several aspects are still not very well understood and are subject of research in progress.

\section{References:}

1. I.E. Locci, P.S. Khadkikar, R.D. Noebe, K. Vedula: Proc. 12th ICEM, Vo1. 4. p. $944(1990)$

2. V.V. Martynov, K. Enami, L.G. Khandros, A.V. Tkachenko, S. Nenno: Scripta Met. 17, 1167 (1983)

3. K. Enami, S. Nenno, K. Shimizu: Trans. JIM 14, 161 (1973)

4. Y.K. Au, C.M. Wayman: Scripta Met. 6,1209 (1972)

5. K. Enami, S. Nenno: Metallurgical Transactions 2, 1487 (1971)

6. S. Rosen, J.A. Goebe1: Trans. Met. Soc. AIME 242, 722 (1968)

7. S. Chakravorty, C.M. Wayman: Metall. Trans. 7A, 569 (1976)

8. K. Enami, J. Hasunuma, A. Nagasawa, S. Nenno: Scripta Met. 10, 879 (1976)

9. M. Chandrasekaran, K. Mukherjee: Mater. Sci. Eng. 13,197 (1974)

10. M. Chandrasekaran, K. Mukherjee: Mater. Sci. Eng. 14, 97 (1974)

11. M. Chandrasekaran, L. Delaey: J, de Physique C4 supplement 12, 43, C4-661 (1982)

12. F. Reynaud: Scripta Met. 11, 765 (1977)

13. K. Enami, A. Nagasawa, S. Nenno: Scripta Met. 12, 223 (1978)

14. L.E. Tanner, D. Schryvers, S.M. Shapiro: Mat. Sci. Eng. A127, 205 (1990)

15. D. Schryvers, L.E. Tanner: Proc. 12th ICEM, Vo1. 4. p. 946 (1990)

16. L.E. Tanner: A.R. Pelton, G. van Tende1oo, D. Schryvers, M.E. Wa11: Scripta Met. 24. 1731 (1990)

17. F. Reynaud: J. Appl. Cryst. 9, 263 (1976)

18. I.M. Robertson, C.M. Wayman: Metallography 17, 43 (1984)

19. V.S. Litvinov, A.A. Arkhangel'skaya: Fiz. Metal. Metalloved. 43, 1044 (1977)

20. K. Enami, S. Nenno: Trans. JIM 19, 571 (1978)

21. M.F. Singleton, J.L. Murray, P. Nash: Binary alloy phase diagrams (ed. T.B. Massalski, ASM, 1986) p. 1421.

22. D. Schrijvers, L.E. Tanner: Micron and Microscopica, 20, 153 (1989)

23. L. Tóth, J. Beyer, J.H. Maas: To be published at ICOMAT-92. 